chronic diseases. Most often PA is self-reported while measures of the aerobic capacity are more seldom measured in subjects with chronic pain.

Objectives: To describe physical activity levels (self-reported and aerobic capacity) in people with chronic pain classified as regional or widespread and to compare the findings with a group that report no pain.

Methods: From the 2016 follow-up of the Swedish population based Epipain cohort ( $n$ 1321), 146 subjects were invited to a clinical assessment where the aerobic capacity was assessed by using a submaximal bicycle test, the EkblomBak test, together with assessment of the Borg scale for perceived exertion (RPE). Aerobic capacity was also classified as low, average or high according to data from the general population. Self-reported physical activity was coded as MVPA $_{\text {rec }}$ if recommended levels of PA was reported (physically active on a moderate level $>150 \mathrm{~min} /$ week (MPA) or on an vigorous level $>75 \mathrm{~min} / \mathrm{week}$ (VPA) or not). The Fear Avoidance Beliefs Questionnaire for PA (FABQ-PA, 0-24 best to worst) and for work (FABQ-W, 0-48 best to worst) were also assessed. The participants were classified as having chronic widespread pain (CWP), chronic regional pain (CRP) or no chronic pain (NCP) based on a pain mannequin presenting $0-18$ pain regions and if pain had lasted for 3 months or more. Chi2 and Kruskal-Wallis tests were performed to study differences between the three pain groups.

Results: $141 / 146$ (97\%) subjects (mean (SD) age 59.4 (8.2) years) whereof $61 \%$ were women, could be classified into pain groups; 43 as CWP (84\% women), 43 as CRP (42\% women) and 55 as NCP (58\% women). The group with CWP was slightly older than those with CRP (mean (SD) age 57.0 (7.6) years vs. 61.9 (6.9) years, p 0.02). The CWP group also had lower aerobic capacity (mean (SD) 2.2 (0.5) l/min vs. 2.6 (0.6) l/min, p 0.03), and a larger proportion was classified as having low aerobic capacity (CWP $21 \%$, CRP $7 \%$ and NCP $10 \%$, p 0.04). The proportion of MVPA $_{\text {rec }}$ did not differ between the groups; CWP $70 \%$, CRP $81 \%$ and NCP $74 \%$ ( $p$ 0.5). There was neither a difference between the groups in BMI, RPE or in sitting hours/week ( $p>0.6)$. However, differences were found in the FABQ where in the PA scale those with CRP had worse scores compared with NCP (mean (SD) 11.2 (7.3) vs. 6.0 (6.0), $\mathrm{p}<0.001$ ), the difference between CWP (mean (SD) $8.9(6.7))$ and NCP was $p$ 0.06. In the work subscale of FABQ, CWP had worse scores compared with CRP (mean (SD) 18.9 (15.7) vs. 10.0 (12.5), p 0.002) and CRP had worse scores compared to those with NCP (mean (SD) 10.0 (12.5) vs. 6.5 (9.1), $\mathrm{p}<0.001)$

Conclusions: In this sample of subjects with chronic pain or no pain, having widespread pain tended to affect the aerobic capacity negatively while self-reports of reaching recommended levels of physical activity did not differ between groups. Fear avoidance in relation to physical activity and especially in relation to work was more noticeable in subjects with chronic pain compared to those with no pain. Measures of aerobic capacity and information of fear avoidance beliefs might help health professionals to better tailor the non-pharmacological treatment for subjects with chronic pain.

Disclosure of Interest: None declared

DOI: 10.1136/annrheumdis-2018-eular.6170

\section{SAT0738-HPR FACTORS ASSOCIATED WITH RISK OF FALLING IN ADULTS WITH KNEE OSTEOARTHRITIS: A CROSS- SECTIONAL STUDY}

D.G. Manlapaz, P. Jayakaran, G. Sole, C.M. Chapple. Centre for Health, Activity and Rehabilitation Research, School of Physiotherapy, University of Otago, Dunedin, New Zealand

Background: There is evidence of increasing number of falls in adults with knee osteoarthritis (OA). However, the contributing factors for falling in adults with knee OA has not been substantially investigated.

Objectives: This cross-sectional study aimed to explore the relationship between falling in adults with knee OA and clinical characteristics of knee OA such as balance, pain, instability, muscle strength, and physical function.

Methods: Participants with knee OA were recruited from the community (Dunedin, New Zealand). The protocol of the study was registered in Australia New Zealand Clinical Trial Registry (ACTRN 12617000154303). Falls characteristics in the preceding year were collected to distinguish between those with and without history of falling. All participants completed the following measures: Sensory Organisation Test (SOT) using NeuroCom SMART Equitest system, version 8.4.0 which produced Composite Score; Knee injury and Osteoarthritis Outcome Score for knee OA related symptoms; Knee outcome survey for self-reported knee instability (buckling); Nicholas MMT hand-held dynamometer for quadriceps and hamstrings isometric muscle strength measured at 20 and 70 degrees; and Timed-Up and Go (TUG) test for physical function.

Results: Sixty-three participants with knee OA (30 female, 33 male), with a mean age (SD) of 53.78 (16.17) years were included in the study. Thirty-one (49\%) participants reported at least one fall in the previous 12 months. The independent ttest suggested that the SOT Composite Score in fallers was significantly less (mean \pm SD: faller $=72.16 \pm 3.26$, non-faller $=74.84 \pm 4.77 ; p=0.012$ ) and the TUG test was significantly longer (mean $\pm S D$ : faller $=7.64 \pm 1.29$, non-faller $=6.74 \pm 0.78$; $p=0.001$ ) when compared with the non-faller group. Also, lower muscle strength of knee flexors and extensors were significantly less in the faller group $(p<0.05)$. Falling in the previous 12 months was associated with Composite Score (OR 0.85 $95 \% \mathrm{Cl} 0.74-0.97, \mathrm{p}=0.017$ ), knee extensors strength [20 degrees (OR 0.76 $95 \% \mathrm{Cl} 0.66-0.82, \mathrm{p}=0.025$ ) and 70 degrees (OR $0.71,95 \% \mathrm{Cl} 0.55-0.91$ $\mathrm{p}=0.008)$ ], and TUG test (OR 2.65, 95\% $\mathrm{Cl} 1.32-5.31, \mathrm{p}=0.006$ ) using univariate logistic regression analysis. There were no changes in these results with multivariable analyses adjusting for age, gender, and body mass index.

Conclusions: The study demonstrated that balance, knee muscle strength, and performance of physical function significantly differed between those with and without the history of falling. An understanding of these risk factors may help in implementing an appropriate evaluation and intervention strategy to reduce falls in this patient population. Given the prevalence of falls in knee OA, this study suggests that falls assessment should be part of the clinical practice routine when evaluating patients with knee $O A$.

Acknowledgements: This study was funded by Mark Steptoe Grant-in-Aid, University of Otago School of Physiotherapy

Disclosure of Interest: None declared

DOI: 10.1136/annrheumdis-2018-eular.4388

\section{SAT0739-HPR OCCUPATIONAL EXPOSURE TO PESTICIDES INCREASES THE RISK OF RHEUMATOID ARTHRITIS: RESULTS FROM THE MALAYSIAN POPULATION-BASED CASE-CONTROL STUDY}

C.L. Too ${ }^{1,2}$, L.K. $\operatorname{Tan}^{3}$, A.F. Nurul Aain ${ }^{1}$, I.S. Lau ${ }^{4}$, M.L. Nor Asiah ${ }^{5}$, S. Salsabil ${ }^{6}$, H. Heselynn ${ }^{7}$, S. Nor Shuhaila ${ }^{7}$, S. Wahinuddin ${ }^{8}$, S.C. Gun ${ }^{9}$, B. Eashwary ${ }^{7}$, M. S. Mohd Shahrir ${ }^{10}$, M. Ainon ${ }^{11}$, R. Azmillah ${ }^{4}$, O. Muhaini ${ }^{12}$, B. Camilla ${ }^{13}$, L. Padyukov ${ }^{2}$, L. Alfredsson ${ }^{13}$, L. Klareskog ${ }^{2}$, M.L. Shahnaz ${ }^{14} .{ }^{1}$ Allergy and Immunology Research Center, Institute for Medical Research, Jalan Pahang, Malaysia; ${ }^{2}$ Department of Medicine, Rheumatology Unit, Karolinska Institutet and Karolinska University Hospital, Stockholm, Sweden; ${ }^{3}$ Immunogenetic Unit, Allergy and Immunology Research Center, Institute for Medical Research, Institute for Medical Research, Jalan Pahang; ${ }^{4}$ Department of Medicine, Selayang Hospital, Selangor, ${ }^{5}$ Institute of Public Health; ${ }^{6}$ Allergy and Immunology Research Center, Institute for Medical Research, Kuala Lumpur, ${ }^{7}$ Department of Medicine, Putrajaya Hospital, Wilayah Persekutuan; ${ }^{8}$ Faculty of Medicine, Universiti Kuala Lumpur Royal College of Medicine Perak, Ipoh; ${ }^{9}$ Department of Medicine, Hospital Tunku Ja'afar Seremban, Seremban; ${ }^{10}$ Faculty of Medicine, Universiti Kebangsaan Malaysia Medical Center, Wilayah Persekutuan; ${ }^{11}$ Department of Medicine, Tengku Ampuan Afzan Hospital, Pahang; ${ }^{12}$ Department of Medicine, Hospital Raja Perempuan Bainun, Perak, Malaysia; ${ }^{13}$ Institute of Environmental Medicine, Karolinska Institutet, Stockholm, Sweden; ${ }^{14}$ Level 12th, Block E7, Parcel E, Federal Government Administrative Center, Ministry of Health Malaysia, Kuala Lumpur, Malaysia

Background: Several studies have suggested farming occupation with exposure to pesticides as risk factor for rheumatoid arthritis (RA)

Objectives: We investigated the association between pesticides exposure and risk of RA subsets in the Malaysian population.

Methods: Data from the Malaysian Epidemiological Investigation of Rheumatoid Arthritis (MyEIRA) population-based case-control study involving 1055 early RA cases and 1057 age, sex and residential area matched controls were analysed. All study subjects answered a structured questionnaire on a broad range of issues including occupational exposures to pesticides. The self-reported information on ever/never occupationally exposed to pesticides was used to estimate the risk of developing ACPA-positive and ACPA-negative RA. Association between pesticides exposure and the HLA-DRB1 shared epitope (SE) was evaluated.

Results: The proportion of ACPA positivity in the RA patients was $64.4 \%$ and $1.9 \%$ in the normal controls. The presence of HLA-DRB1 SE alleles in RA patients was $40.2 \%$ and $15.8 \%$ in the normal controls. Our data demonstrated that occupational exposure to pesticides was significantly associated with an increased risk of developing RA in the Malaysian population (OR 2.31, 95\% Cl 1.12-4.73, $p=0.03$ ) The association between occupational exposure to pesticides and risk of RA was observed with ACPA-positive RA (OR 3.10 95\% Cl 1.49-6.47, p=0.003), but not with ACPA-negative RA. A dramatically increased risk for ACPA-positive RA was seen in individuals who both exposed to pesticides occupationally and carried SE alleles (OR 28.06, 95\% Cl 3.58-220.09, p<0.0001).

Conclusions: This study demonstrates that occupational exposure to pesticides is associated with an increased risk of ACPA-positive RA in Malaysian population.

\section{REFERENCES:}

[1] Sverdrup B, Källberg H, Bengtsson C, Lundberg I, Padyukov L, Alfredsson $L$, Klareskog $L$ and the Epidemiological Investigation of Rheumatoid Arthritis study group. Association between occupational exposure to mineral oil and rheumatoid arthritis: results from the Swedish EIRA case-control study- Arthritis Research \& Therapy 2005;7:R1296-R1303. 